\title{
Thin topological insulator film in a perpendicular magnetic field
}

\author{
A. A. Zyuzin ${ }^{1}$ and A. A. Burkov ${ }^{1,2}$ \\ ${ }^{1}$ Department of Physics and Astronomy, University of Waterloo, Waterloo, Ontario N2L 3G1, Canada \\ ${ }^{2}$ Department of Physics, California Institute of Technology, Pasadena, California 91125, USA \\ (Received 14 March 2011; revised manuscript received 31 March 2011; published 6 May 2011)
}

\begin{abstract}
We report on a study of an ultrathin topological insulator film with hybridization between the top and bottom surfaces, placed in a quantizing perpendicular magnetic field. We calculate the full Landau-level spectrum of the film as a function of the applied magnetic field and the magnitude of the hybridization matrix element, taking into account both the orbital and the Zeeman spin splitting effects of the field. For an undoped film, we find a quantum phase transition between a state with a zero Hall conductivity and a state with a quantized Hall conductivity equal to $e^{2} / h$, as a function of the magnitude of the applied field. The transition is driven by the competition between the Zeeman and the hybridization energies.
\end{abstract}

DOI: 10.1103/PhysRevB.83.195413

PACS number(s): 73.50.-h, 73.43.Nq

\section{INTRODUCTION}

The topological insulator (TI) is a new phase of matter, which has recently been discovered in materials, long known and extensively studied in the fields of traditional semiconductor physics $(\mathrm{HgTe})$ and the physics of thermoelectrics $\left(\mathrm{Bi}_{2} \mathrm{Se}_{3}\right.$ and $\left.\mathrm{Bi}_{2} \mathrm{Te}_{3}\right) .{ }^{1-3}$ Somewhat uncharacteristically of the "standard" order of things in condensed-matter physics, the TI phase was first predicted to occur in these materials theoretically ${ }^{1}$ and only later seen experimentally. ${ }^{2}$ One obvious reason for this is that the main experimental manifestation of the TI phase, namely the occurrence of metallic edge states in insulating samples, is a somewhat subtle effect, unlike, e.g., the spectacular quantization of the Hall conductivity in a close relative of the TI, the two-dimensional electron gas (2DEG) in the quantum Hall effect (QHE) regime. While theoretically the TI phase can in fact be characterized by a quantized physical quantity, the topological magnetoelectric susceptibility ${ }^{4,5}$ measuring this quantity experimentally is not straightforward. Currently, the only realistic proposal involves measurement of the magneto-optical response in a thin TI film when the time-reversal symmetry is broken, with the Faraday and Kerr rotation angles predicted to acquire universal values in this case. ${ }^{6-8}$

As far as transport phenomena in the TI are concerned, theoretical effort has mostly been concentrated on studying the effects of the characteristic spin-momentum locking of the helical TI surface states, which has an obvious potential in spintronics. ${ }^{9-18}$ An important practical issue, relevant to all transport studies of the TI, is that all currently known TI materials, while theoretically insulators, in reality are metallic in the bulk. This happens due to unavoidable doping, introduced by impurities and crystal lattice defects. One possible way to deal with the problem is to grow TI samples in the form of thin films. ${ }^{19-22}$ This allows one to both directly reduce the bulk contribution to transport by simply reducing the bulk volume and to control the carrier concentration in the bulk through gating. ${ }^{23-25}$

Ultrathin TI films also have interesting physical properties, such as the above-mentioned universal magneto-optical response, ${ }^{6-8}$ possible excitonic superfluidity, ${ }^{26}$ strongly improved thermoelectric performance, ${ }^{27}$ quantum spin Hall ${ }^{28,29}$ and quantum anomalous Hall (QAH) effects, ${ }^{30}$ which have so far been explored only theoretically. Of particular interest are the effects that arise in the ultrathin limit, when the top and bottom surfaces of a TI film start to hybridize. ${ }^{21}$ We have recently pointed out ${ }^{31}$ that magnetic response, in particular response to field, applied in the plane of the film, is highly nontrivial in this limit, with the film undergoing a topological insulator to semimetal quantum phase transition as a function of the field.

In this paper we study the properties of TI thin films in a perpendicular quantizing magnetic field. We calculate the full Landau-level (LL) spectrum of a TI film with hybridization between the top and bottom surfaces and the Hall conductivity. One would normally expect the Hall conductivity to be zero in an undoped charge-neutral film, which is indeed what happens when hybridization is stronger than the Zeeman splitting. As the magnetic field is increased, however, we find a quantum phase transition at which the Hall conductivity jumps from zero to $e^{2} / h$. The transition happens when the Zeeman energy becomes larger than the hybridization energy and is shown here to be a direct consequence of the well-known characteristic feature of the Dirac-fermion LL spectrum, namely the existence of a zero-energy LL. The transition we find is analogous to the transition to the QAH state discussed in Ref. 30 in the context of TI films, doped with magnetic impurities. Here we demonstrate that the same physics can be realized in a more straightforward and currently experimentally accessible way, simply utilizing an applied magnetic field. Indeed, experimental work on magnetotransport properties of thin TI films in quantizing perpendicular magnetic field has already appeared in the literature. ${ }^{32-35}$

\section{LANDAU-LEVEL SPECTRUM OF AN ULTRATHIN TI FILM}

We start from the following Hamiltonian of an ultrathin TI film in the presence of a perpendicular magnetic field:

$$
\begin{aligned}
\mathcal{H}= & \int d \mathbf{r} \Psi^{\dagger}(\mathbf{r})\left[v_{F} \tau^{z}(\hat{z} \times \boldsymbol{\sigma}) \cdot\left(-i \nabla+\frac{e}{c} \mathbf{A}\right)\right. \\
& \left.+\Delta_{z} \sigma^{z}+\Delta_{t} \tau^{x}\right] \Psi(\mathbf{r}) .
\end{aligned}
$$


Here $v_{F}$ is the surface Dirac cone Fermi velocity, $\Delta_{z}=$ $g \mu_{B} B / 2$ is the Zeeman energy, associated with the applied magnetic field $\mathbf{B}=B \hat{z}$ (we will assume $B \geqslant 0$ henceforth), $\Delta_{t}$ is the hybridization matrix element (we will also assume $\Delta_{t} \geqslant 0$ ), and $\hbar=1$ units are used. We have introduced Pauli matrices $\sigma$ and $\tau$ to describe the real spin and the surface pseudospin degrees of freedom and suppressed the explicit spin and pseudospin indices for clarity of notation.

To diagonalize Eq. (1), we choose the Landau gauge for the vector potential $\mathbf{A}=x B \hat{y}$ and define LL ladder operators in the standard way as

$$
a=(\ell / \sqrt{2})\left(\pi_{x}-i \pi_{y}\right), \quad a^{\dagger}=(\ell / \sqrt{2})\left(\pi_{x}+i \pi_{y}\right),
$$

where $\ell=\sqrt{c / e B}$ is the magnetic length and $\pi=-i \nabla+$ $(e / c) \mathbf{A}$ is the kinetic momentum. The single-particle Hamiltonian operator can then be written in terms of the ladder operators as

$$
H=\frac{i \omega_{B}}{\sqrt{2}} \tau^{z}\left(\sigma^{+} a-\sigma^{-} a^{\dagger}\right)+\Delta_{z} \sigma^{z}+\Delta_{t} \tau^{x},
$$

where we have introduced a characteristic frequency $\omega_{B}=$ $v_{F} / \ell$, which plays a role analogous to the cyclotron frequency in the LL spectrum of a regular 2DEG.

It is clear from Eq. (3) that the single-particle eigenstates have the following general form:

$$
\begin{aligned}
|n \alpha s\rangle= & u_{n T \uparrow}^{\alpha s}|n-1, T, \uparrow\rangle+u_{n T \downarrow}^{\alpha s}|n, T, \downarrow\rangle \\
& +u_{n B \uparrow}^{\alpha s}|n-1, B, \uparrow\rangle+u_{n B \downarrow}^{\alpha s}|n, B, \downarrow\rangle .
\end{aligned}
$$

Here $|n, T(B), \uparrow(\downarrow)\rangle$ is the $n$th LL eigenstate on the top (bottom) surface with spin up (down), $\alpha=0,1$ and $s= \pm$ label the four eigenstates of Eq. (3), corresponding to each LL index $n=0, \ldots, \infty$, and $u_{n}^{\alpha s}$ are the corresponding complex fourcomponent spinor wave functions. We have also suppressed the intra-LL orbital label for brevity. The physical meaning of the $\alpha$ and $s$ indices will become clear shortly.

The problem of finding the eigenstates of Eq. (3) thus reduces to diagonalizing the following $4 \times 4$ matrix:

$$
\left(\begin{array}{cccc}
\Delta_{z} & -i \omega_{B} \sqrt{2 n} & \Delta_{t} & 0 \\
i \omega_{B} \sqrt{2 n} & -\Delta_{z} & 0 & \Delta_{t} \\
\Delta_{t} & 0 & \Delta_{z} & i \omega_{B} \sqrt{2 n} \\
0 & \Delta_{t} & -i \omega_{B} \sqrt{2 n} & -\Delta_{z}
\end{array}\right) .
$$

Diagonalizing Eq. (5), we find the following LL spectrum:

$$
\epsilon_{n \alpha \pm}=(-1)^{\alpha} \sqrt{2 \omega_{B}^{2} n+\left(\Delta_{z} \pm \Delta_{t}\right)^{2}} .
$$

The corresponding spinor wave functions are given by

$$
u_{n}^{\alpha s}=\left[i s(-1)^{\alpha} f_{n \alpha s_{+}},-s f_{n \alpha s_{-}}, i(-1)^{\alpha} f_{n \alpha s+}, f_{n \alpha s_{-}}\right],
$$

where

$$
f_{n \alpha s \pm}=\frac{1}{2} \sqrt{1 \pm \frac{\Delta_{z}+s \Delta_{t}}{\epsilon_{n \alpha s}}} .
$$

The LL spectrum thus consists of two sets: electronlike $\left(\epsilon_{n 0 \pm}\right)$ and holelike $\left(\epsilon_{n 1 \pm}\right)$ Landau levels. Within each set, every LL is further split into a doublet $( \pm)$. Note that this splitting results from the presence of both the Zeeman spin splitting and the hybridization, and vanishes if any one of them is zero (except for the $n=0 \mathrm{LL}$, which is split whenever $\Delta_{t}$ is nonzero).

The $n=0$ LL are special. As seen from Eq. (4), electrons in these levels are fully spin polarized, i.e., only the spin-down states are occupied. Correspondingly, the $n=0$ level is only split into two sublevels, unlike all other LLs, which are split into four. This is the well-known "zero-mode anomaly" of the Dirac-fermion LL spectrum, ${ }^{36-38}$ which will play an important role in our story.

Explicitly, the two $n=0$ LL wave functions are given by

$$
u_{0}^{0-}=\frac{1}{\sqrt{2}}(0,1,0,1), \quad u_{0}^{1+}=\frac{1}{\sqrt{2}}(0,-1,0,1),
$$

when $\Delta_{z}<\Delta_{t}$. The corresponding LL energies in this case are

$$
\epsilon_{00-}=\left|\Delta_{z}-\Delta_{t}\right|, \quad \epsilon_{01+}=-\left|\Delta_{z}+\Delta_{t}\right| .
$$

On the other hand, when $\Delta_{z}>\Delta_{t}, u_{0}^{0-}$ is replaced by $u_{0}^{1-}$,

$$
u_{0}^{1-}=\frac{1}{\sqrt{2}}(0,1,0,1),
$$

with the corresponding energy given by

$$
\epsilon_{01-}=-\left|\Delta_{z}-\Delta_{t}\right| \text {. }
$$

Thus one of the two $n=0$ sublevels moves from the set of electronlike levels into the set of holelike levels. This change of the character of the LL spectrum as a function of $\Delta_{z}$ is illustrated in Fig. 1. As we demonstrate below, the transition to purely holelike $n=0 \mathrm{LL}$ is manifested in a jump of the Hall conductivity at fixed chemical potential $\epsilon_{F}=0$ by $e^{2} / h$.

\section{HALL CONDUCTIVITY}

Given the above LL spectrum, we can now evaluate the Hall conductivity of the TI film. Assuming the Fermi level to be always in a gap between the LLs, the Kubo-formula expression for the Hall conductivity reads

$$
\begin{aligned}
\sigma_{x y}= & \frac{\omega_{B}^{2} e^{2}}{2 \pi} \sum_{n \alpha s \neq n^{\prime} \alpha^{\prime} s^{\prime}} \operatorname{Im}\left[\left\langle n \alpha s\left|\tau^{z} \sigma^{y}\right| n^{\prime} \alpha^{\prime} s^{\prime}\right\rangle\right. \\
& \left.\times\left\langle n^{\prime} \alpha^{\prime} s^{\prime}\left|\tau^{z} \sigma^{x}\right| n \alpha s\right\rangle\right] \frac{n_{F}\left(\epsilon_{n \alpha s}\right)-n_{F}\left(\epsilon_{n^{\prime} \alpha^{\prime} s^{\prime}}\right)}{\left(\epsilon_{n \alpha s}-\epsilon_{n^{\prime} \alpha^{\prime} s^{\prime}}\right)^{2}},
\end{aligned}
$$

where $n_{F}$ is the Fermi-Dirac distribution (we will assume zero temperature in all calculations). The matrix elements in Eq. (13) are nonzero only when $s=s^{\prime}$ and $n^{\prime}=n \pm 1$. Evaluating the matrix elements, we obtain the following expression for the Hall conductivity:

$$
\begin{aligned}
\sigma_{x y}= & \frac{e^{2}}{4 \pi} \sum_{n=0}^{\infty} \sum_{\alpha=0,1} \sum_{s= \pm}\left\{(2 n+1)\left[n_{F}\left(\epsilon_{n \alpha s}\right)-n_{F}\left(\epsilon_{n+1 \alpha s}\right)\right]\right. \\
& \left.+\left(\Delta_{z}+s \Delta_{t}\right)\left[\frac{n_{F}\left(\epsilon_{n+1 \alpha s}\right)}{\epsilon_{n+1 \alpha s}}-\frac{n_{F}\left(\epsilon_{n \alpha s}\right)}{\epsilon_{n \alpha s}}\right]\right\} .
\end{aligned}
$$

The first term in Eq. (14) corresponds to the well-known expression for the Dirac-fermion Hall conductivity. Assuming $n$ electronlike LLs are filled and neglecting the Zeeman and 


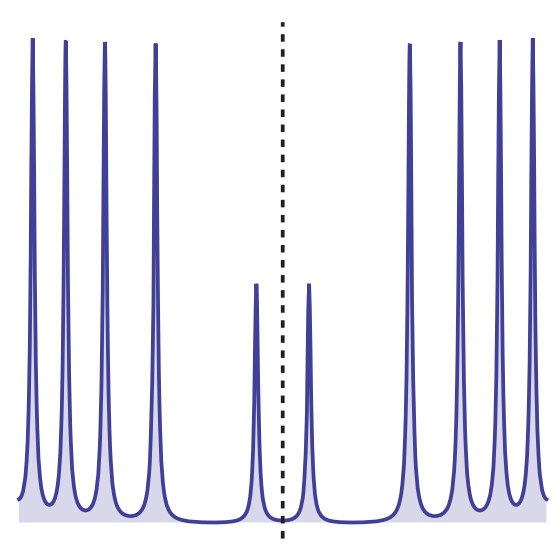

(a)

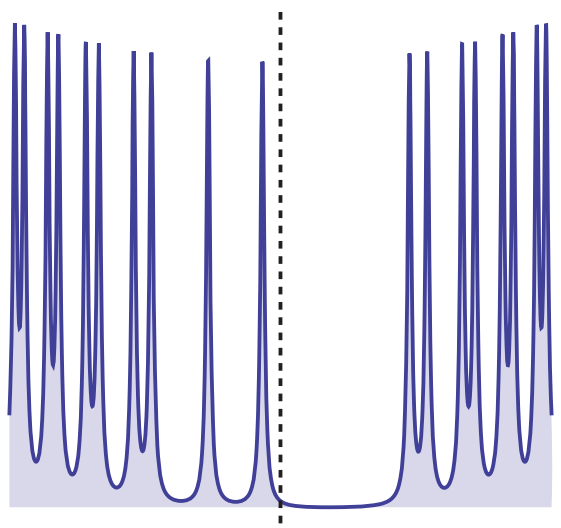

(b)

FIG. 1. (Color online) LL density of states of a thin TI film (broadening added by hand). Dashed line represents the zero of energy, set at the Dirac point position in the absence of the magnetic field. (a) $\Delta_{z}=0, \Delta_{t}>0$ case. Only the $n=0 \mathrm{LL}$ is split and the spectrum is particle-hole symmetric. (b) $\Delta_{z}>\Delta_{t}>0$ case. Both $n=0 \mathrm{LL}$ are holelike and particle-hole symmetry of the spectrum is broken. All LL splittings in the figure are exaggerated relative to the main LL spacing.

hybridization splittings, which are negligible for all $n>0 \mathrm{LLs}$, this term gives

$$
\sigma_{x y}=\frac{e^{2}}{h}(2 n+1), n \geqslant 1,
$$

where we have restored $\hbar$ for clarity. This result is the same (up to a factor of $1 / 2$, accounting for half the degeneracy) as the well-known result for the Hall conductivity of graphene. ${ }^{39}$

The contribution of the $n=0 \mathrm{LL}$ is contained in the second term in Eq. (14), which also contains the physics unique to TI thin films. The effect of this term is most easily seen at the point $\epsilon_{F}=0$, which corresponds to the charge-neutrality point in the limit $\Delta_{z}=0\left(\Delta_{z}>0\right.$ breaks particle-hole symmetry, as seen from Fig. 1). In this case we obtain the following expression for the Hall conductivity (note again that $\Delta_{z}, \Delta_{t} \geqslant 0$ ):

$$
\sigma_{x y}=\frac{e^{2}}{2 h}\left[\operatorname{sgn}\left(\Delta_{z}-\Delta_{t}\right)+1\right] .
$$

Thus one can see that $\sigma_{x y}$, evaluated at fixed Fermi energy $\epsilon_{F}=0$, jumps from $0\left(\Delta_{z}<\Delta_{t}\right)$ to $\pm e^{2} / h\left(\Delta_{z}>\Delta_{t}\right)$ as a function of the applied magnetic field. This transition is the result of the change of the character of the $n=0$ doublet of LL, which happens as a function of $\Delta_{z} / \Delta_{t}$, and which was described in Sec. II. To reiterate, when $\Delta_{z}<\Delta_{t}$, one of the $n=0$ LLs $\left(u^{0-}\right)$ is electronlike, while the other one $\left(u^{1+}\right)$ is holelike. When $\Delta_{z}>\Delta_{t}$, both $n=0$ LLs become holelike $\left(u^{1 \pm}\right)$, and the Hall conductivity thus increases by $e^{2} / h$. This change in the value of the Hall conductivity is of course only observed when the Fermi level is initially within the hybridization gap of the $n=0$ LLs.

It is important to note that the above transition at a fixed Fermi energy is associated with a small increase of the (two-dimensional) electron density by $\delta n_{e} \sim 1 /\left.2 \pi \ell^{2}\right|_{\Delta_{z}=\Delta_{t}}=$ $m \Delta_{t} / \pi$, since the high-field side of the transition $\Delta_{z}>\Delta_{t}$ differs from the low-field side by an extra filled LL. If the charge density is held fixed and one starts from the low-field limit when the Fermi level is in the hybridization gap between the two $n=0$ Landau sublevels, no transition occurs upon increasing the field, as the Fermi level moves with the field and always stays in the gap. However, the fixed Fermi energy situation might in fact be more physically relevant for a sample in a transport-measurement setup, attached to external conducting leads (admittedly, this is a subtle issue and what actually happens may depend on details of the experimental setup and various characteristics of the sample).

\section{DISCUSSION AND CONCLUSIONS}

In this paper we have studied the properties of an ultrathin TI film, in which the top and bottom surfaces are hybridized, placed in a perpendicular magnetic field. We have calculated the LL spectrum of such a film, taking into account the Zeeman spin splitting. We have also evaluated the Hall conductivity of the film and demonstrated that the Hall conductivity has a nontrivial dependence on the ratio of two energy scales: the Zeeman energy $\Delta_{z}$ and the hybridization energy $\Delta_{t}$. Namely, we have shown that a thin film with hybridization between the top and bottom surfaces undergoes a quantum phase transition from a "trivial insulator" state, in which the Hall conductivity at charge neutrality is zero to a "Hall insulator" state, in which the Hall conductivity is equal to $e^{2} / h$. The transition happens when $\Delta_{z}=\Delta_{t}$ and is associated with a nonanalytic contribution to the Hall conductivity, which comes entirely from the $n=0$ LLs. The transition is accompanied by a change in the character of the $n=0$ LLs. On the low-field side of the transition one of the $n=0$ sublevels is electronlike, while the second one is holelike. On the high-field side both LL are holelike.

Finally, let us briefly discuss experimental observability of the proposed effect. To this end we estimate the energy scales, associated with the TI film LLs. Taking $B \sim 10 \mathrm{~T}$, we obtain $\Delta_{z} \sim 1 \mathrm{meV}$ (using $g \sim 1$ for a very conservative estimate), and $\omega_{B} \sim 10 \mathrm{meV}$. Assuming $\Delta_{t} \sim \Delta_{z}$ (which is needed to observe the phase transition we have described), we then have $\omega_{B} \gg \Delta_{t}, \Delta_{z}$. Thus observing the "trivial insulator" to "Hall insulator" phase transition might require more stringent conditions than a general observation of the QHE in this system would require. One needs low, i.e., less than about $10 \mathrm{~K}$, temperatures and a clean enough sample that a $\sim 1 \mathrm{meV}$ hybridization gap between the two $n=0$ LLs is not washed out by disorder. However, recent experiments actually suggest a much larger value of the $g$ factor for the surface states in 
$\mathrm{Bi}_{2} \mathrm{Se}_{3}$ and related materials, ${ }^{32,33}$ as large as $g \approx 50$ (which is not unexpected due to the strong spin-orbit interactions in the bulk material ${ }^{40}$ ), and this makes the proposed phase transition much more easily observable. But even the most stringent conditions, required if $g \sim 1$, are in fact standard in semiconductor 2DEG QHE measurements and thus should be easily achievable, at least for molecular beam epitaxy grown TI films.

\section{ACKNOWLEDGMENTS}

We acknowledge useful discussions with J. Alicea, L. Balents, J. Eisenstein, and O. Motrunich. Financial support was provided by the NSERC of Canada and a University of Waterloo start-up grant. A.A.B. gratefully acknowledges hospitality of the Caltech Physics Department, where part of this work was performed.
${ }^{1}$ C. L. Kane and E. J. Mele, Phys. Rev. Lett. 95, 146802 (2005); B. A. Bernevig, T. L. Hughes, and S.-C. Zhang, Science 314, 1757 (2006); J. E. Moore and L. Balents, Phys. Rev. B 75, 121306 (2007); L. Fu, C. L. Kane, and E. J. Mele, Phys. Rev. Lett. 98, 106803 (2007); R. Roy, Phys. Rev. B 79, 195322 (2009); H. Zhang, C.-X. Liu, X.-L. Qi, X. Dai, Z. Fang, and S.-C. Zhang, Nat. Phys. 5, 438 (2009).

${ }^{2}$ Y. Xia, D. Qian, D. Hsieh, L. Wray, A. Pal, H. Lin, A. Bansil, D. Grauer, Y. S. Hor, R. J. Cava, and M. Z. Hasan, Nat. Phys. 5, 398 (2009).

${ }^{3}$ M. Z. Hasan and C. L. Kane, Rev. Mod. Phys. 82, 3045 (2010); X.-L. Qi and S.-C. Zhang, e-print arXiv:1008.2026 (to be published); M. Z. Hasan and J. E. Moore, Ann. Rev. Cond. Mat. Phys. 2, 55 (2011); J. E. Moore, Nature (London) 464, 194 (2010).

${ }^{4}$ X.-L. Qi, T. L. Hughes, and S.-C. Zhang, Phys. Rev. B 78, 195424 (2008).

${ }^{5}$ A. M. Essin, J. E. Moore, and D. Vanderbilt, Phys. Rev. Lett. 102, 146805 (2009).

${ }^{6}$ W.-K. Tse and A. H. MacDonald, Phys. Rev. Lett. 105, 057401 (2010).

${ }^{7}$ W.-K. Tse and A. H. MacDonald, Phys. Rev. B 82, 161104(R) (2010).

${ }^{8}$ J. Maciejko, X.-L. Qi, H. D. Drew, and S.-C. Zhang, Phys. Rev. Lett. 105, 166803 (2010).

${ }^{9}$ S. Raghu, S. B. Chung, X.-L. Qi, and S.-C. Zhang, Phys. Rev. Lett. 104, 116401 (2010).

${ }^{10}$ I. Garate and M. Franz, Phys. Rev. Lett. 104, 146802 (2010).

${ }^{11}$ I. Garate and M. Franz, Phys. Rev. B 81, 172408 (2010).

${ }^{12}$ S. Mondal, D. Sen, K. Sengupta, and R. Shankar, Phys. Rev. Lett. 104, 046403 (2010).

${ }^{13}$ S. Mondal, D. Sen, K. Sengupta, and R. Shankar, Phys. Rev. B 82, 045120 (2010).

${ }^{14}$ T. Yokoyama, Y. Tanaka, and N. Nagaosa, Phys. Rev. B 81, 121401(R) (2010).

${ }^{15}$ T. Yokoyama, J. Zang, and N. Nagaosa, Phys. Rev. B 81, 241410(R) (2010).

${ }^{16}$ A. A. Burkov and D. G. Hawthorn, Phys. Rev. Lett. 105, 066802 (2010).

${ }^{17}$ D. Culcer, E. H. Hwang, T. D. Stanescu, and S. Das Sarma, Phys. Rev. B 82, 155457 (2010).

${ }^{18}$ K. Nomura and N. Nagaosa, Phys. Rev. B 82, 161401 (2010).

${ }^{19}$ G. Zhang, H. Qin, J. Teng, J. Guo, Q. Guo, X. Dai, Z. Fang, and K. Wu, Appl. Phys. Lett. 95, 053114 (2009).
${ }^{20}$ H. Peng, K. Lai, D. Kong, S. Meister, Y. Chen, X.-L. Qi, S.-C. Zhang, Z.-X. Shen, and Y. Cui, Nat. Mater. 9, 225 (2010).

${ }^{21}$ Y. Zhang, K. He, C.-Z. Chang, C.-L. Song, L.-L. Wang, X. Chen, J.-F. Jia, Z. Fang, X. Dai, W.-Y. Shan, S.-Q. Shen, Q. Niu, X.-L. Qi, S.-C. Zhang, X.-C. Ma, and Q.-K. Xue, Nat. Phys. 6, 584 (2010).

${ }^{22}$ J. Linder, T. Yokoyama, and A. Sudbø, Phys. Rev. B 80, 205401 (2009).

${ }^{23}$ H. Steinberg, D. R. Gardner, Y. S. Lee, and P. Jarillo-Herrero, Nano Lett. 10, 5032 (2010).

${ }^{24}$ J. G. Checkelsky, Y. S. Hor, R. J. Cava, and N. P. Ong, e-print arXiv:1003.3883 (to be published).

${ }^{25}$ J. Chen, H. J. Qin, F. Yang, J. Liu, T. Guan, F. M. Qu, G. H. Zhang, J. R. Shi, X. C. Xie, C. L. Yang, K. H. Wu, Y. Q. Li, and L. Lu, Phys. Rev. Lett. 105, 176602 (2010).

${ }^{26}$ B. Seradjeh, J. E. Moore, and M. Franz, Phys. Rev. Lett. 103, 066402 (2009).

${ }^{27}$ P. Ghaemi, R. S. K. Mong, and J. E. Moore, Phys. Rev. Lett. 105, 166603 (2010).

${ }^{28}$ H.-Z. Lu, W.-Y. Shan, W. Yao, Q. Niu, and S.-Q. Shen, Phys. Rev. B 81, 115407 (2010).

${ }^{29}$ C.-X. Liu, H. J. Zhang, B. Yan, X.-L. Qi, T. Frauenheim, X. Dai, Z. Fang, and S.-C. Zhang, Phys. Rev. B 81, 041307(R) (2010).

${ }^{30}$ R. Yu, W. Zhang, H.-J. Zhang, S.-C. Zhang, X. Dai, and Z. Fang, Science 329, 61 (2010).

${ }^{31}$ A. A. Zyuzin, M. D. Hook, and A. A. Burkov, e-print arXiv:1102.0349 (to be published).

${ }^{32}$ J. G. Analytis, R. D. McDonald, S. C. Riggs, J.-H. Chu, G. S. Boebinger, and I. R. Fisher, Nat. Phys. 6, 960 (2010).

${ }^{33}$ Z. Ren, A. A. Taskin, S. Sasaki, K. Segawa, and Y. Ando, Phys. Rev. B 82, 241306(R) (2010).

${ }^{34}$ J. Xiong, A. C. Petersen, D. Qu, R. J. Cava, and N. P. Ong, e-print arXiv:1101.1315 (to be published).

${ }^{35}$ B. Sacépé, J. B. Oostinga, J. Li, A. Ubaldini, N. J. G. Couto, E. Giannini, and A. F. Morpurgo, e-print arXiv:1101.2352 (to be published).

${ }^{36}$ R. Jackiw, Phys. Rev. D 29, 2375 (1984).

${ }^{37}$ G. W. Semenoff, Phys. Rev. Lett. 53, 2449 (1984).

${ }^{38}$ F. D. M. Haldane, Phys. Rev. Lett. 61, 2015 (1988).

${ }^{39}$ V. P. Gusynin and S. G. Sharapov, Phys. Rev. Lett. 95, 146801 (2005).

${ }^{40}$ H. Kohler and E. Wochner, Phys. Status Solidi B 67, 665 (1975). 